\title{
A large-scale analysis of targeted metabolomics data from heterogeneous biological samples provides insights into metabolite dynamics
}

\author{
Ho-Joon Lee ${ }^{1,2,3,4} \mathbb{D} \cdot$ Daniel M. Kremer ${ }^{1} \cdot$ Peter Sajjakulnukit $^{1} \cdot$ Li $^{\text {Zhang }}{ }^{1,5} \cdot$ Costas A. Lyssiotis $^{1,4,6} \mathbb{C}^{\mathbb{D}}$
}

Received: 25 January 2019 / Accepted: 20 June 2019 / Published online: 9 July 2019

(c) The Author(s) 2019

\begin{abstract}
Introduction We previously developed a tandem mass spectrometry-based label-free targeted metabolomics analysis framework coupled to two distinct chromatographic methods, reversed-phase liquid chromatography (RPLC) and hydrophilic interaction liquid chromatography (HILIC), with dynamic multiple reaction monitoring (dMRM) for simultaneous detection of over 200 metabolites to study core metabolic pathways.

Objectives We aim to analyze a large-scale heterogeneous data compendium generated from our LC-MS/MS platform with both RPLC and HILIC methods to systematically assess measurement quality in biological replicate groups and to investigate metabolite abundance changes and patterns across different biological conditions.

Methods Our metabolomics framework was applied in a wide range of experimental systems including cancer cell lines, tumors, extracellular media, primary cells, immune cells, organoids, organs (e.g. pancreata), tissues, and sera from human and mice. We also developed computational and statistical analysis pipelines, which include hierarchical clustering, replicategroup CV analysis, correlation analysis, and case-control paired analysis.

Results We generated a compendium of 42 heterogeneous deidentified datasets with 635 samples using both RPLC and HILIC methods. There exist metabolite signatures that correspond to various phenotypes of the heterogeneous datasets, involved in several metabolic pathways. The RPLC method shows overall better reproducibility than the HILIC method for most metabolites including polar amino acids. Correlation analysis reveals high confidence metabolites irrespective of experimental systems such as methionine, phenylalanine, and taurine. We also identify homocystine, reduced glutathione, and phosphoenolpyruvic acid as highly dynamic metabolites across all case-control paired samples.

Conclusions Our study is expected to serve as a resource and a reference point for a systematic analysis of label-free LC-MS/ MS targeted metabolomics data in both RPLC and HILIC methods with dMRM.
\end{abstract}

Keywords Targeted metabolomics $\cdot$ LC-MS/MS $\cdot$ RPLC $\cdot$ HILIC $\cdot$ Measurement reliability $\cdot$ Amino acids $\cdot$ Metabolite dynamics

\section{Introduction}

Electronic supplementary material The online version of this article (https://doi.org/10.1007/s11306-019-1564-8) contains supplementary material, which is available to authorized users.

Daniel M. Kremer and Peter Sajjakulnukit contributed equally to this study.

Ho-Joon Lee

esavant@med.umich.edu

Costas A. Lyssiotis

clyssiot@med.umich.edu

Extended author information available on the last page of the article
Mass spectrometry (MS) is a popular and powerful platform for metabolomics studies (Johnson et al. 2016; Patti et al. 2012; Wishart 2016). Although nuclear magnetic resonance (NMR)-based metabolomics is also widely used, MS is more easily coupled to various chromatographic columns to separate analytes prior to analysis, thereby reducing the complexity of a biological sample and increasing sensitivity for simultaneous detection of a large number of metabolites (Griffiths et al. 2010; Zhou and Yin 2016).

MS-based metabolomics is performed predominantly by subjecting samples to chromatographic separation before 
MS analysis. Chromatographic columns make it possible to separate complex analyte mixtures based on physicochemical properties of a wide range of compounds, including isomers. Liquid chromatography (LC) is most frequently used, while gas chromatography (GC) is preferred for measuring volatile compounds. LC methods include reversed-phase liquid chromatography (RPLC) and hydrophilic interaction liquid chromatography (HILIC). RPLC is typically used for a broad range of metabolites, especially nonpolar and weakly polar metabolites, whereas HILIC has a complementary usage for hydrophilic, polar, and ionic metabolites such as sugars, amino acids, and nucleic acids (Buszewski and Noga 2012; Cubbon et al. 2010; Ivanisevic et al. 2013; Lu et al. 2008; Rojo et al. 2012; Tang et al. 2016). While a recent study performed a systematic technical evaluation of hydrophilic and hydrophobic LC-MS in metabolomics profiling (Xie et al. 2018), it lacks a biological evaluation of abundance measurements in biological replicates under a wide range of different conditions.

We previously developed a tandem mass spectrometrybased targeted metabolomics system that profiles abundance of more than 200 metabolites as a steady-state snapshot of global metabolism. It utilizes both RPLC and HILIC methods with dynamic MRM (dMRM) as a means to maximize the coverage and sensitivity of target metabolites. Together with our customized computational and statistical analysis pipelines, this system has been recently applied in several biological contexts (Halbrook et al. 2018; Schofield et al. 2018; Sousa et al. 2016; Svoboda et al. 2018). Herein, we report heterogeneous data sets generated from a wide range of experiments and sample types using our LC-MS/MS targeted metabolomics platforms over a period of about 1 year from 2017 to 2018 . The data were collected with no specific criteria to both be unbiased and include as much data as possible. Using these, we carried out comprehensive global meta-analysis in which we systematically evaluated data quality of both RPLC and HILIC methods by statistical measures and characterized global patterns of metabolite changes and variability in case versus control groups.

\section{Materials and methods}

\subsection{Sample preparation}

Preparation of the sample types below has been described before (Halbrook et al. 2018; Schofield et al. 2018; Sousa et al. 2016; Svoboda et al. 2018; Yuan et al. 2012). A brief description follows. Each distinct experimental condition has at least three biological replicate samples prepared from different cell culture plates/preparations or animals (i.e., $n \geq 3$ ), which defines a (biological) replicate group in this study.

\subsection{Primary and cultured cells}

Biological replicates with $\mathrm{n} \geq 3$ were seeded at equivalent density, grow in $\log$ phase, and at end point media was fully aspirated. Aqueous metabolites of adherent primary or cultured cells on 6-well or $10 \mathrm{~cm}^{2}$ plates were extracted by adding $1 \mathrm{~mL}$ or $4 \mathrm{~mL}$ of $80 \%$ cold $\left(-80^{\circ} \mathrm{C}\right)$ methanol, respectively, followed by incubation at $-80^{\circ} \mathrm{C}$ for $10 \mathrm{~min}$. Cells were then scrapped and all material, including soluble and insoluble material, was collected. This was then followed by centrifugation at $14,000 \mathrm{rpm}$ at $4{ }^{\circ} \mathrm{C}$ for $10 \mathrm{~min}$ to pellet the insoluble material. Suspension cells were gently centrifuged to pellet and media was completely aspirated. Metabolites were extracted using the method above. The procedure is done on a bucket of dry ice and as quickly as possible in order to stop metabolism immediately. Samples were normalized by the volume corresponding to protein concentration measured from parallelly prepared lysates (typically, 1-2 million cells at 70-80\% confluence). Then, samples were dried under vacuum and suspended in a 1:1 $\mathrm{H}_{2} \mathrm{O}$ /methanol solution for LC-MS analysis.

\subsection{Tissues, organs, and tumors}

Samples of 50-200 mg $(\mathrm{n} \geq 3)$ were placed in a tube containing $1 \mathrm{~mL}$ of $80 \%$ cold $\left(-80{ }^{\circ} \mathrm{C}\right)$ methanol and then homogenized using steel beads and a Qiagen Tissue Lyser by multiple rounds of 45 -second shaking at room temperature before centrifugation at $14,000 \mathrm{rpm}$ at $4{ }^{\circ} \mathrm{C}$ for $10 \mathrm{~min}$. Samples were normalized by taking the volume corresponding to $10 \mathrm{mg}$ of the tumor weight and further processed as above for LC-MS analysis.

\subsection{Cultured media and sera}

Metabolites from equivalent volumes of media or sera $(\mathrm{n} \geq 3$; typically, $\sim 200 \mu \mathrm{L}$ ) were extracted by adding $100 \%$ cold $\left(-80^{\circ} \mathrm{C}\right)$ methanol with a $1: 4$ ratio of the sample to methanol (i.e., $80 \%$ methanol final) and further processed as above for LC-MS analysis.

\subsection{LC-MS/MS metabolomics analysis}

Our LC-MS/MS metabolomics analysis was performed as described previously (Halbrook et al. 2018; Schofield et al. 2018; Sousa et al. 2016). In brief, an Agilent 1290 UHPLC and 6490 Triple Quadrupole (QqQ) mass spectrometer (LC-MS/MS) were used for label-free targeted metabolomics analysis. Agilent MassHunter Optimizer and Workstation Software LC-MS Data Acquisition for 6400 Series Triple Quadrupole B.08.00 was used for standard 
optimization and data acquisition. Agilent MassHunter Workstation Software Quantitative Analysis Version B.0700 for QqQ was used for initial raw data extraction and analysis. Each MRM transition and its retention time of left delta and right delta of $1 \mathrm{~min}$. Additional parameters include mass extraction window of $0.05 \mathrm{Da}$ right and left from the extract $\mathrm{m} / \mathrm{z}$, Agile2 integrator algorithm, peak filter of 100 counts, noise algorithm RMS, noise SD multiplier of $5 \mathrm{~min}, \mathrm{~S} / \mathrm{N}$ 3, Accuracy Max 20\% max \%Dev, and Quadratic/Cubic Savitzky-Golay smoothing algorithm with smoothing function width of 14 and Gaussian width of 5 .

For RPLC, a Waters Acquity UPLC BEH TSS C18 column $(2.1 \times 100 \mathrm{~mm}, 1.7 \mu \mathrm{m})$ was used in the positive ionization mode with mobile phase (A) consisting of $0.5 \mathrm{mM}$ $\mathrm{NH}_{4} \mathrm{~F}$ and $0.1 \%$ formic acid in water; mobile phase (B) consisting of $0.1 \%$ formic acid in acetonitrile. Gradient program: mobile phase (B) was held at $1 \%$ for $1.5 \mathrm{~min}$, increased to $80 \%$ at $15 \mathrm{~min}$, then to $99 \%$ at $17 \mathrm{~min}$ and held for $2 \mathrm{~min}$ before going to initial condition and held for $10 \mathrm{~min}$. For HILIC, a Waters Acquity UPLC BEH amide column $(2.1 \times 100 \mathrm{~mm}, 1.7 \mu \mathrm{m})$ was used in the negative ionization mode with mobile phase (A) consisting of $20 \mathrm{mM}$ ammonium acetate $\left(\mathrm{NH}_{4} \mathrm{OAc}\right)$ in water at $\mathrm{pH} 9.6$; mobile phase (B) consisting of acetonitrile (ACN). Gradient program: mobile phase (B) was held at $85 \%$ for $1 \mathrm{~min}$, decreased to $65 \%$ at $12 \mathrm{~min}$, then to $40 \%$ at $15 \mathrm{~min}$ and held for $5 \mathrm{~min}$ before going to the initial condition and held for $10 \mathrm{~min}$.

Both columns were at $40^{\circ} \mathrm{C}$ and $3 \mu \mathrm{L}$ of each sample was injected into the LC-MS with a flow rate of $0.2 \mathrm{~mL} /$ min. Calibration was achieved through Agilent ESI-Low Concentration Tuning Mix. Optimization was performed on the 6490 QqQ in the RPLC-positive or HILIC-negative mode for each of 245 standard compounds (215 and 217 compounds for RPLC-positive and HILIC-negative, respectively) to obtain the best fragment ion and MS parameters such as fragmentation energy for each standard. Retention time (RT) for each standard was measured from a pure standard solution or a mixture standard solution. The LC-MS/ MS methods were created with dynamic MRM (dMRM) with RTs, RT windows, and transitions of all 245 standard compounds (see Supplementary Table, "Methods"). Key parameters of electrospray ionization (ESI) in both the positive and the negative acquisition modes are: Gas temp 275 ${ }^{\circ} \mathrm{C}$, Gas Flow 14 1/min, Nebulizer at 20 psi, SheathGasHeater $250^{\circ} \mathrm{C}$, SheathGasFlow $11 \mathrm{~L} / \mathrm{min}$, and Capillary $3000 \mathrm{~V}$. For MS: Delta EMV $200 \mathrm{~V}$ or $350 \mathrm{~V}$ for the positive or negative acquisition mode respectively and Cycle Time $500 \mathrm{~ms}$ and Cell Acc $4 \mathrm{~V}$ for both modes. In this study we denote the dMRM method with RPLC in the positive ionization mode by RPLC-Pos-dMRM and the dMRM method with HILIC in the negative ionization mode by HILIC-Neg-dMRM. We note that our methods do not distinguish stereoisomers, hence care should be taken in interpretation of such data.

\subsection{Computational data processing, quality control, and statistical analysis}

Pre-processed data with Agilent MassHunter Workstation Software Quantitative Analysis were post-processed for further quality control in the programming language R. Let $A_{i j}$ be a data matrix of raw abundance with $M$ metabolites and $N$ samples, i.e., $i=1$ to $M$ and $j=1$ to $N$. First, we examined the distribution of sums of all metabolite abundance peak areas across individual samples, $\left\{S_{j}=\sum_{i=1}^{M} A_{i j}: j=1\right.$ to $\left.N\right\}$, in a given experiment as a measure for equal sample loading into the instrument. Any outlier sample was removed, which is defined by a loading difference of greater than $70 \%$ compared to the average of the total abundance sums. The choice of $70 \%$ is based on our experience, rather than an optimization technique, and this has consistently yielded interpretable data and biologically relevant results in all experiments on our platform (Halbrook et al. 2019; Schofield et al. 2018; Svoboda et al. 2018). Next, we calculated coefficients of variation $(\mathrm{CVs})$ in all biological replicate groups $(\mathrm{n} \geq 3)$ for each metabolite given a cut-off value of peak areas in each of the RPLC-Pos-dMRM and the HILIC-Neg-dMRM methods. We then compared distributions of $\mathrm{CVs}$ for the whole dataset for a set of peak area cut-off values of $0,1000,5000$, $10000,15000,20000,25000$ and 30000 in each method. A noise cut-off value of peak areas in each method was chosen by manual inspection of the $\mathrm{CV}$ distributions. The noisefiltered data of individual samples were then normalized by the total intensity of all metabolites. We retained only those metabolites with at least two replicate measurements for a given experimental variable. The remaining missing value in each condition for each metabolite was filled with the median value of the other replicate measurements. Let $A_{i j}^{\prime}\left(i=1\right.$ to $M^{\prime} \leq M, j=1$ to $\left.N^{\prime} \leq N\right)$ be the noise-filtered, normalized, and median-imputed data matrix. Then, each metabolite abundance level in each sample was divided (i.e., scaled) by the mean of all abundance levels across all samples in a given experiment, $A_{i j}^{*}=A_{i j}^{\prime} /\left(\frac{1}{N^{\prime}} \sum_{k=1}^{N^{\prime}} A_{i k}^{\prime}\right)$ (“"normalized relative abundance" hereafter), for comparisons, statistical analyses, and visualizations among metabolites. This normalization and scaling method has been used in our previous studies with biologically meaningful results (Halbrook et al. 2019; Schofield et al. 2018; Svoboda et al. 2018). A comparison analysis with other alternative methods (Kirpich et al. 2018; Li et al. 2017; van den Berg et al. 2006; Wanichthanarak et al. 2017) is beyond the scope of this study. Finally, we visually inspected a correlation heatmap profile of the samples of the resultant data to identify and remove any further outlier samples based on hierarchical clustering and abnormal heatmap patterns. Heatmaps were generated with the function, heatmap.2, from the Bioconductor package, gplots, and hierarchical clustering was 
performed with default parameters in heatmap.2. Pathway analysis was done using the webtool, MetaboAnalyst (Chong et al. 2018).

\section{Results and discussion}

\subsection{Global view of a compendium of heterogeneous targeted metabolomics datasets}

A flowchart overview of our approach in this study is depicted in Fig. 1a. We compiled 42 datasets of LC-MS/ MS-based targeted metabolomics with both RPLC-PosdMRM and HILIC-Neg-dMRM methods. There are a total of 638 samples analyzed, which include cancer cell lines, tumors, extracellular media, primary cells, immune cells, organoids, organs (e.g. pancreata), tissues, sera, and shRNA/drug treatments in human and mice. A total of 448 measurements were made in both methods for 285 unique compound entities (due to multiple detection). We retained measurements for those metabolites detected in both RPLCPos-dMRM and HILIC-Neg-dMRM methods as independent measurements. From this compendium we constructed a 448 by 638 data matrix for global characterization (Supplementary Table S1). $36.7 \%$ of the values in this data matrix were missing, and we removed 11 samples where no compound was measured. We find that this is a generic problem associated with generating metabolomics data in a large number of heterogeneous samples, especially with a HILIC method. It relates to several factors including general metabolite stability (e.g. succinate and fumarate), poor ionization efficiency of some metabolites such as carbohydrates, large dynamic ranges of certain metabolites in different cell/tissue types (e.g. GABA and certain carbohydrates), and complex matrix effects. Figure $1 \mathrm{~b}$ depicts a correlation heatmap and a hierarchical clustering of all metabolites in both RPLC-Pos-dMRM and HILIC-Neg-dMRM methods. Figure 1c shows a correlation heatmap and a hierarchical clustering of all samples. We find that there are a few groups of highly correlated metabolites. For example, at the height of 6 in the dendrogram tree, the 31 metabolites in Cluster \#1 are enriched in purine, pyrimidine, glycine/ serine, and glutathione metabolism, and the 88 metabolites in Cluster \#2 include metabolites in the TCA cycle, glycolysis, pyruvate, and phenylalanine/tyrosine/tryptophan metabolism (Fig. 1d). This suggests that those metabolites tend to change their abundance in the same direction across different conditions, which suggests that they are subject to pathway-level metabolic regulation. The lack of clustering among samples reflects the heterogeneous nature of all datasets and indicates that experimental bias is not driving clustering and influencing downstream analysis. In fact, the most highly correlated 25 samples (Cluster \#3, Fig. 1c) do not exhibit common phenotypes, and this cluster includes cell lines, murine tumors and several experimentally distinct variables. Therefore, it suggests the existence of general metabolic phenotypes that can arise from various perturbations, independent of cell/tissue types.

\subsection{Analysis of normalized relative abundance}

Raw data from the LC-MS/MS analysis are peak areas of ion counts for each identified metabolite. These raw data do not reflect absolute abundance and cannot be compared directly between experiments run at different times. Instead we normalized each sample by the total ion counts of all metabolites to approximately correct equal sample loading. Then, each metabolite abundance value was divided by the mean of all abundance values across all samples in each experiment, defined as normalized relative abundance, i.e., $A_{i j}^{*}$ (see Sect. 2.3 of Materials and Methods). We first examined normalized relative abundance distributions of all metabolites across all datasets using medians in replicategroup measurements. Figure 2a shows the number of median measurements for each metabolite across all 183 replicate groups. There are a total of 13,026 measurements for 448 metabolites in both RPLC-Pos-dMRM and HILIC-NegdMRM methods in the 42 experiments. 71 metabolites were measured in all 183 replicate groups (Fig. 2a). All of these came from the RPLC-Pos-dMRM method. This may indicate that the RPLC-Pos-dMRM method is more robust in detecting those metabolites than the HILIC-Neg-dMRM method. Those most frequently detected metabolites across different conditions are enriched in metabolism of amino acids, nitrogen, glutathione, and purine (MetaboAnalyst; FDR $<0.01)$. The remaining metabolites show a monotonic decrease in the number of measured replicate groups. It is unclear what is the generating function or mechanism of this linearly decreasing distribution.

We next calculated the average of all abundance values for each metabolite. Figure $2 b$ shows a histogram of the average abundance values for all 448 metabolites and Fig. 2c shows a distribution of the ordered average abundance values. Three of the top 20 metabolites are involved in cysteine/methionine metabolism (cystine, acetyl-serine, and glutathione) and others in glycolysis, hexosamine, amino acids, mevalonate, energy, and redox metabolism. We also find that one of the top 20 metabolites, phenylalanine, is among the most reproducible metabolites by both RPLC-Pos-dMRM and HILIC-Neg-dMRM methods, along with methionine and taurine, as discussed below (Sect. 3.4). On the other hand, the bottom 20 metabolites include GDP-glucose, NADP+, fumaric acid, glycerol, dihydrofolate, leucine, docosahexaenoic acid, homocystine, TMP, sucrose, and 
(A)

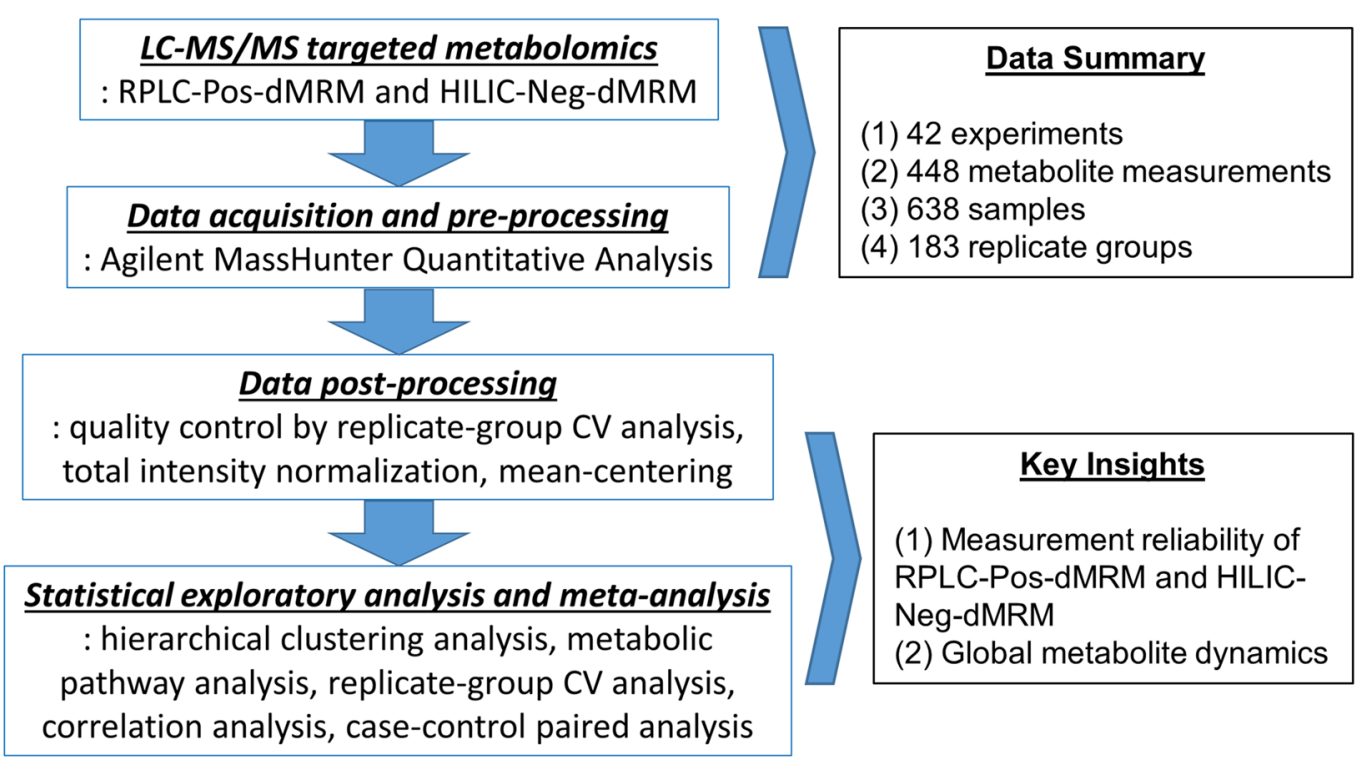

(B)
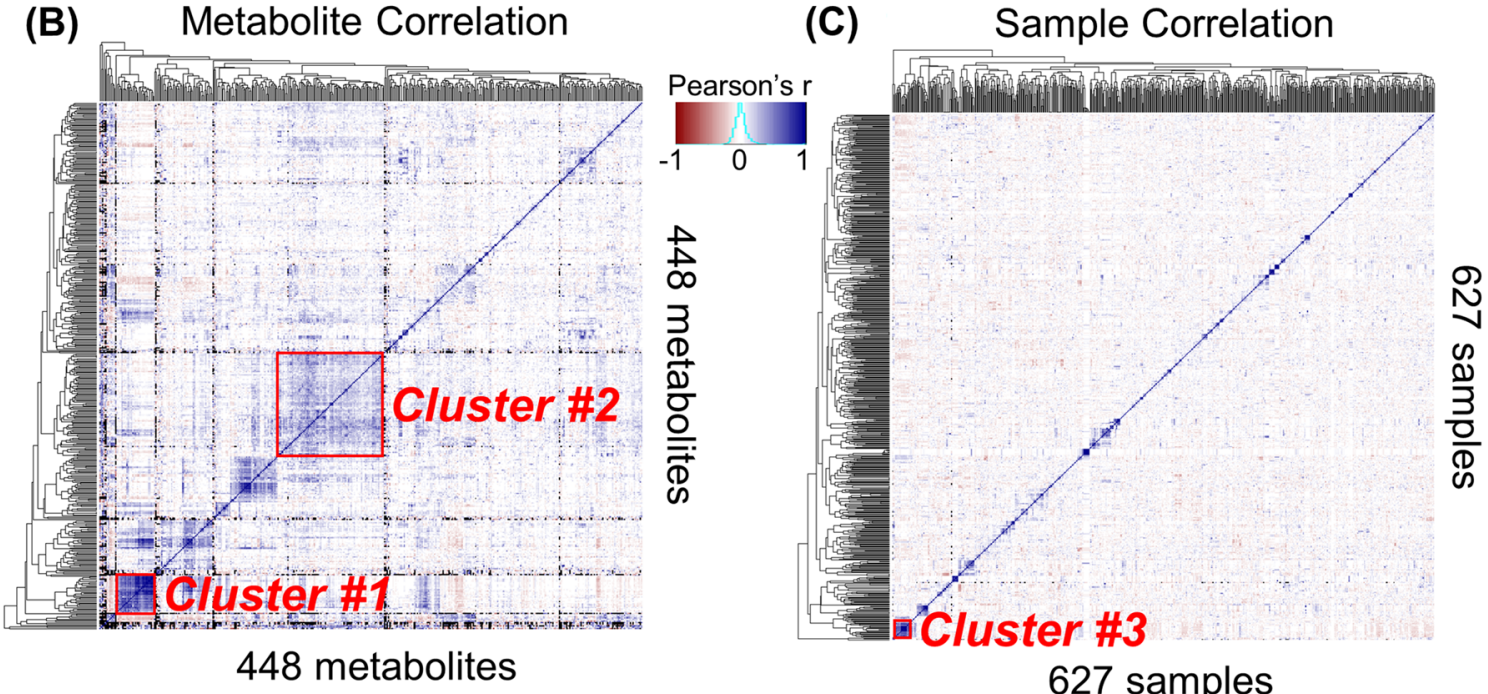

(RPLC-Pos-dMRM and HILIC-Neg-dMRM)
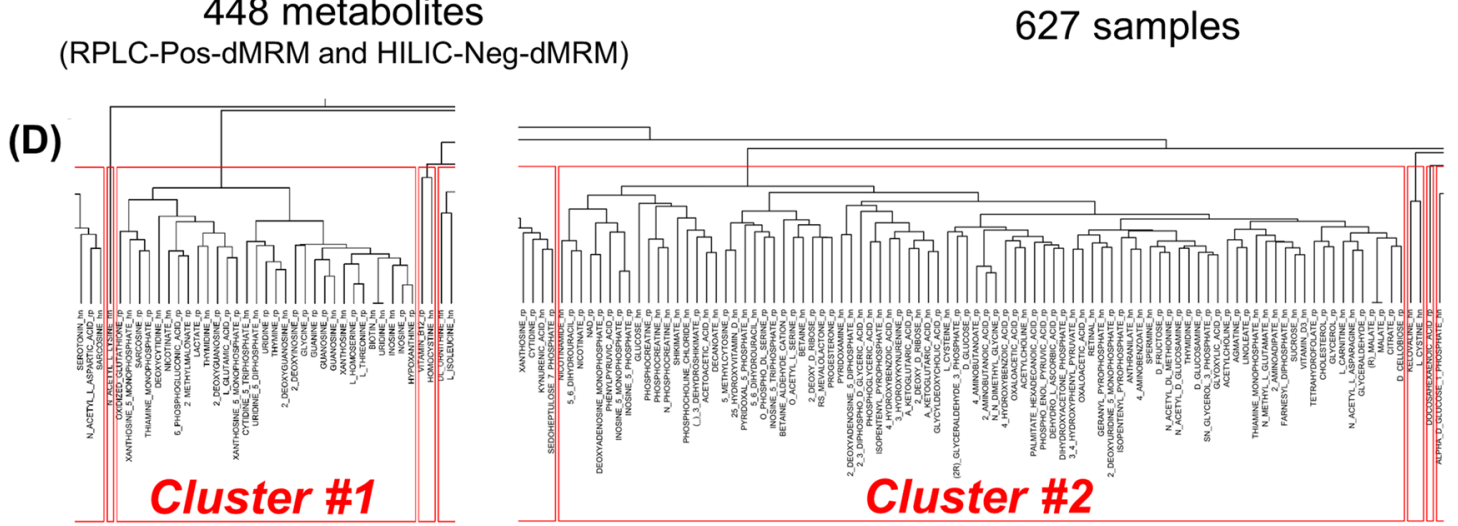

Fig. 1 Global view of the metabolomics data compendium. a A flowchart and a summary of our approach. b A correlation heatmap of 448 metabolite measurements of relative normalized abundance from both RPLC-Pos-dMRM and HILIC-Neg-dMRM methods along with unsupervised hierarchical clustering. The color key is based on Pearson correlation coefficients. c A correlation heatmap of 627 samples along with unsupervised hierarchical clustering. The color code is the same as in b. See Supplementary Table S1 for the data used for b, c. d Dendrogram trees of Cluster \#1 and Cluster \#2 at the height of 6. The suffix, "rp", in the metabolite names stands for RPLC-PosdMRM and "hn" for HILIC-Neg-dMRM 

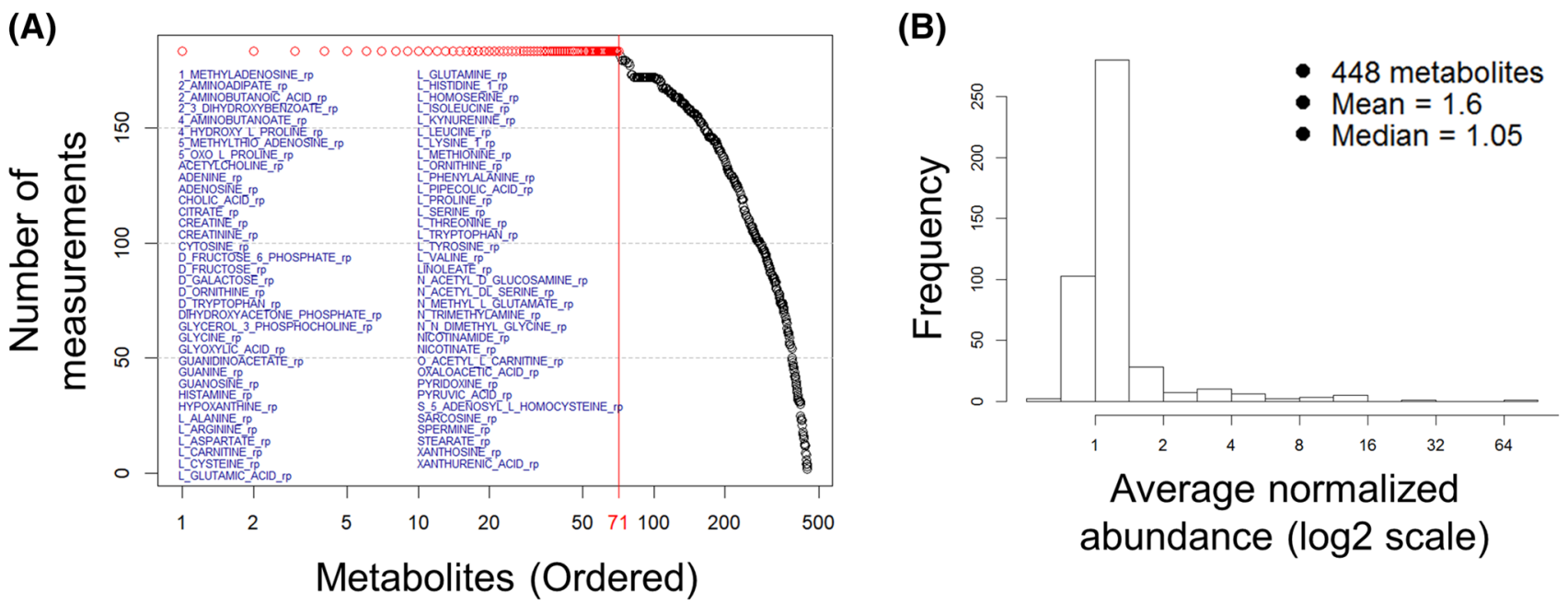

(C)
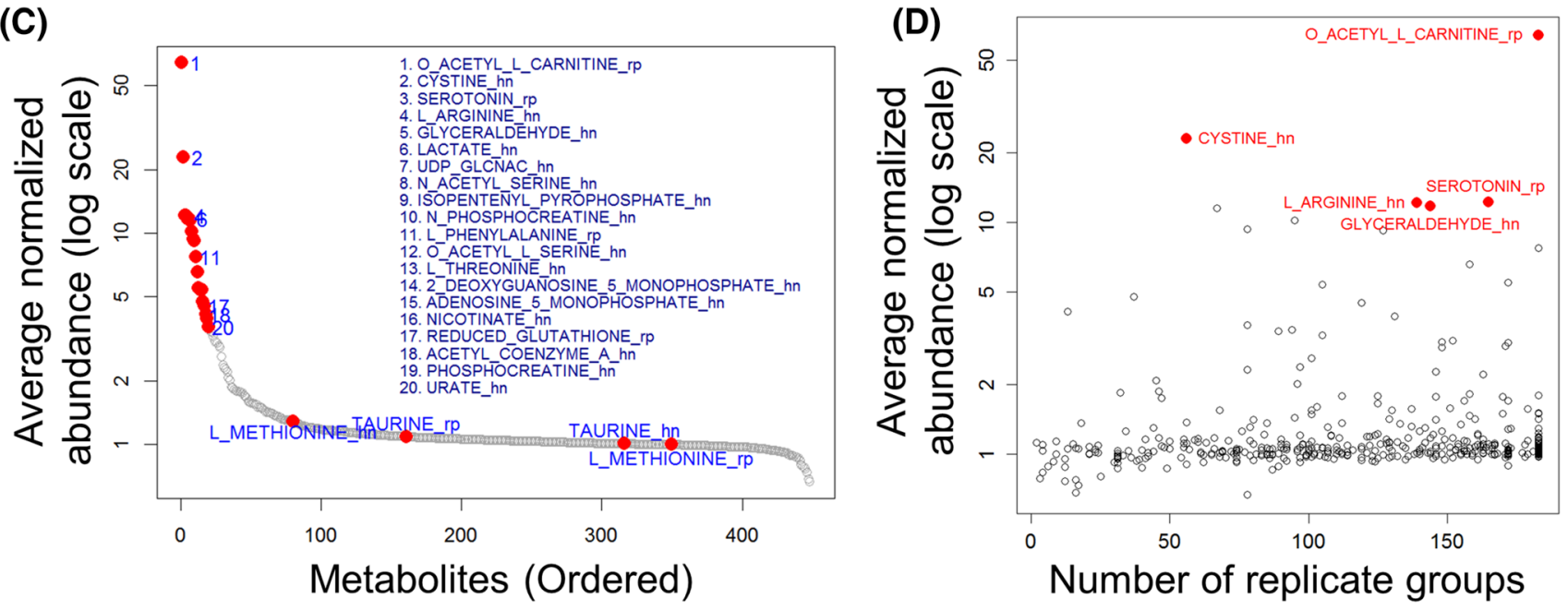

Fig. 2 Analysis of relative abundance. a The distribution of the numbers of median measurements for 448 metabolites across all 183 biological replicate groups. There are 71 metabolites with measurements in the maximum 183 replicate groups as listed and as indicated in red circles. See also Supplementary Table S2 for the list of 71 metabolites. b A histogram distribution of the average normalized abundance

deoxyadenosine. These metabolites are involved in many different metabolic pathways with no significant enrichment (MetaboAnalyst), suggesting that low abundance is not a pathway-specific or pathway-level feature. Figure $2 \mathrm{~d}$ is a scatter plot of the average abundance and the number of replicate groups with measurements. The top five metabolites are highly abundant in 56-183 distinct groups on average, indicating the tendency of more frequent detection of more abundant metabolites. This analysis offers insight into the importance of those relatively high abundant metabolites in diverse conditions with potential effects on phenotypic differences. values for all 448 metabolites. c An ordered distribution of $\mathbf{b}$. The top 20 metabolites are listed along with methionine and taurine (cf. Figure 4F). See also Supplementary Table S2 for the list of the top 20 metabolites. d A scatter plot of the average normalized abundance of b or $\mathbf{c}$ and the numbers of replicate groups with measurements. The top 5 highly abundant metabolites are shown in red

\subsection{Replicate-group CV analysis of RPLC-Pos-dMRM and HILIC-Neg-dMRM data}

The coefficient of variation or $\mathrm{CV}$ (=SD/mean) is a standard quantitative measure of statistical dispersion or variability (Carobene et al. 2013). We use CV to assess variability or consistency of replicate measurements in each replicate group for quality assessment. We calculated biological replicate-group CVs for both the RPLC-Pos-dMRM and HILIC-Neg-dMRM methods and examined their quality or reliability differences. Figure 3 a shows a comparison of replicate-group $\mathrm{CV}$ distributions of the two methods. There are 
28,765 CV values in RPLC-Pos-dMRM and 22,069 CV values in HILIC-Neg-dMRM from all 183 replicate groups for 220 and 228 metabolites, respectively. RPLC-Pos-dMRM measurements show more CVs $<0.2$ than HILIC-NegdMRM measurements by about two-fold. The summary statistics also show overall better quality of RPLC-Pos-dMRM measurements than HILIC-Neg-dMRM. The reliability of each metabolite measurement was assessed by the average $\mathrm{CV}$ in all replicate groups. We denote the average $\mathrm{CV}$ of a metabolite by $\overline{C V_{k}}=\frac{1}{m} \sum_{i=1}^{m} C V_{k, i}$, where $k$ is a metabolite and $m$ is the number of replicate groups with measurements. The ordered distributions of $\left\{\overline{C V_{k}}\right\}$ in Fig. 3b shows an overview of average measurement reliability of all metabolites in each method (see also Supplementary Table S3). We calculated $\overline{C V_{k}}$ for only those metabolites that were measured in at least 20 replicate groups $(m \geq 20)$, which yielded 215 and 210 metabolites from RPLC-Pos-dMRM and HILICNeg-dMRM, respectively, with 154 of them in common. The reliability difference between the two methods is very clear, although Pearson's correlation of CVs from the two methods for the 154 common metabolites shows a significant positive relationship $(r=0.37 ; p<2.4 \mathrm{e}-6)$. This suggests greater chromatographic regularity or compound stability in RPLCPos-dMRM than HILIC-Neg-dMRM, which is consistent with previous studies on HILIC (Hao et al. 2008; Rhoades and Weljie 2016). It also suggests that most metabolites show a similar tendency of reliability in each method. The quantity, $\overline{C V_{k}}$, from our heterogeneous dataset could be used as a reliability index or a guidance in each method for other similar applications of LC-MS/MS targeted metabolomics. Among the 50 most reliable metabolites from each method, there are 20 overlapping metabolites including leucine/isoleucine, methionine, hydroxyproline, valine, nicotinamide, glutamate, phenylalanine, serine, glycine, and NAD (see Supplementary Table S3), which are likely to exhibit high stability and efficient ionizations in both methods. Among the 50 least reliable metabolites from each method, there are 14 overlapping metabolites including GDP-glucose, uridine 5'-diphosphate, cytidine 5'-diphosphate, sucrose, malate, guanosine 5'-diphosphate, lactate, and glyceraldehyde (see Supplementary Table S3), which would require more careful measurements and interpretations.

To examine the reliability in more detail, we further restricted our focus to those metabolites with measurements in at least $70 \%$ of the 183 replicate groups (i.e., CV missing values less than $30 \%$ or $m \geq 183 \times 0.7=128$ ) for statistical robustness. There are 145 such metabolites for RPLC-Pos-dMRM and 77 such metabolites for HILIC-NegdMRM. The heatmaps and hierarchical clustering in Fig. 3c, $\mathrm{d}$ show groups of metabolites with low CVs (i.e., better reliability; rows in blue) in each method. While we visually notice overall better reliability in RPLC-Pos-dMRM from the heatmaps, there are metabolites with low CVs across all replicate groups in HILIC measurements such as taurine, thymine, phenylalanine, hypotaurine, pyridoxate, methionine, and tryptophan.

A pathway analysis of most reliable metabolites with $\overline{C V_{k}}<0.4$ (125 and 54 metabolites in RPLC-Pos-dMRM and HILIC-Neg-dMRM, respectively) shows that each method does not favor any unique pathway. Both methods tend to have reliable measurements for amino acids and nucleotides biosynthesis pathways, the main difference being the number of reliable metabolites given a threshold of $\overline{C V_{k}}$. This pathway analysis supports the aforementioned positive correlation of $\left\{\overline{C V_{k}}\right\}$ between the two methods. On the other hand, by focusing on 19 amino acids from both methods, we find that four amino acids show lower $\overline{C V_{k}}$ in HILIC-Neg-dMRM than in RPLC-Pos-dMRM: phenylalanine, tryptophan, proline, and asparagine, the first three of which show similarly good measurement reproducibility as hydrophobic in both methods with $\overline{C V_{k}}<0.4$ (Fig. 3e, f). This suggests that those three amino acids may be used as most reliable reference metabolites in abundance measurements as well as in LC-MS/MS method development in both chromatographic column conditions. It is also observed that asparagine, aspartate, and cysteine are less reliable in both methods (Fig. 3e, f). We note that phenylalanine and tryptophan are often used as quality control standards in metabolomics laboratories on an empirical basis, corroborating our findings (Dr. Maureen Kachman, personal communication). We point out that all polar amino acids except asparagine show better reproducibility (i.e., lower $\overline{C V_{k}}$ ) in RPLC-Pos-dMRM than HILICNeg-dMRM (Fig. 3e, f).

\subsection{Correlation analysis of RPLC-Pos-dMRM and HILIC-Neg-dMRM data}

We continued to examine differences between RPLC-PosdMRM and HILIC-Neg-dMRM from a correlation point of view by focusing on those metabolites that were measured in at least $70 \%$ of all 42 experiments with both methods. We asked if abundance profiles of each metabolite from the two methods were well correlated in individual experiments as they were conducted on different days. A total of 3601 measurements were made for 237 metabolites in the 42 experiments. There were 221 metabolites that were measured in at least two experiments with both methods. 47 of them were measured in at least $70 \%$ of all 42 experiments. For each metabolite in each experiment, we calculated the Pearson correlation coefficient between two abundance profiles measured by the two methods. Figure 4 a shows a heatmap of all RPLC-HILIC correlation coefficients, where the metabolites were sorted by the average correlation across all the experiments and the columns were sorted by the average correlation across all the metabolites. Larger circles in darker blues indicate good correlation between the two 
(A) Replicate-group CV distributions

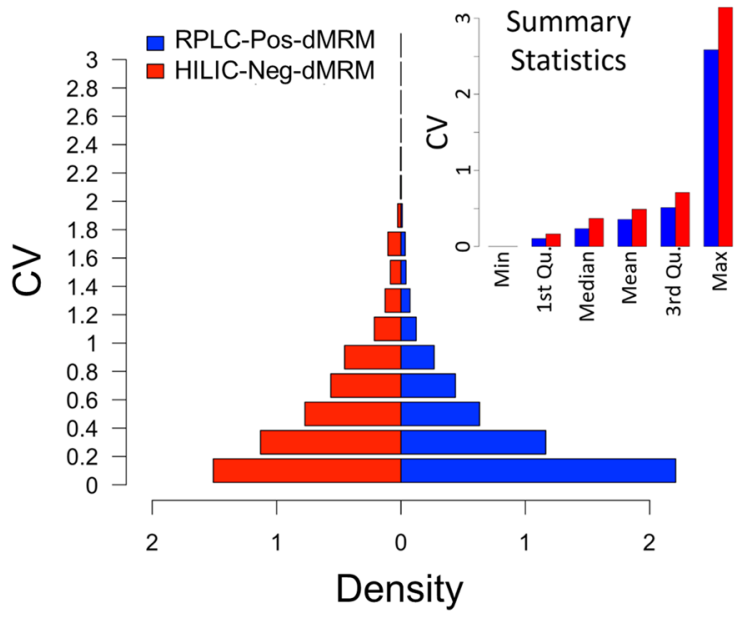

(B) Average CV Distributions

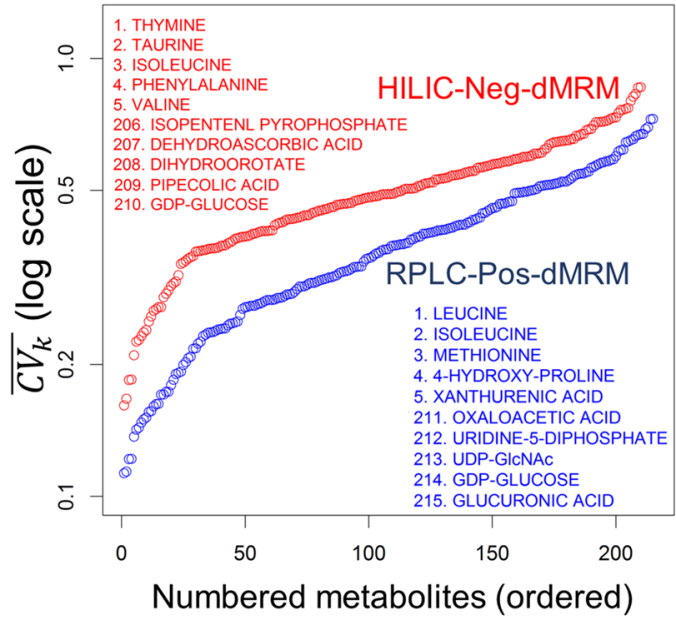

(C) RPLC-Pos-dMRM

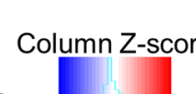

HILIC-Neg-dMRM

(D)

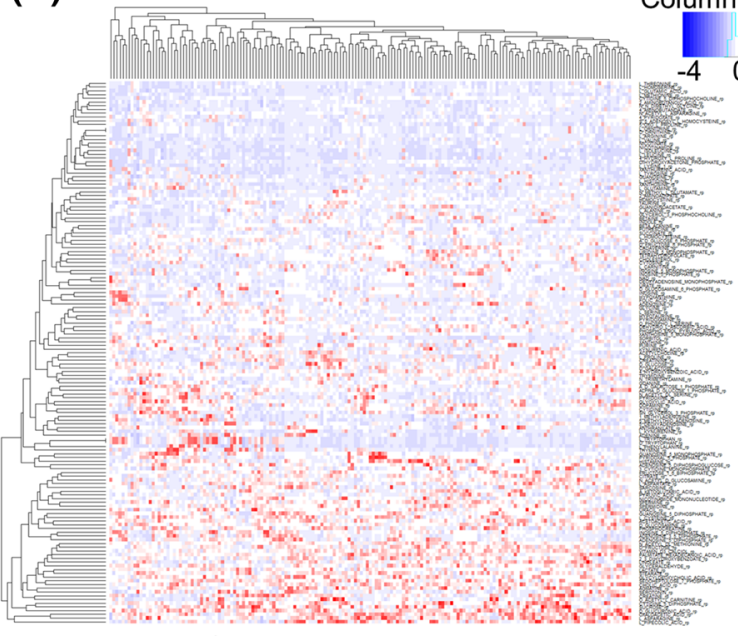

173 replicate groups
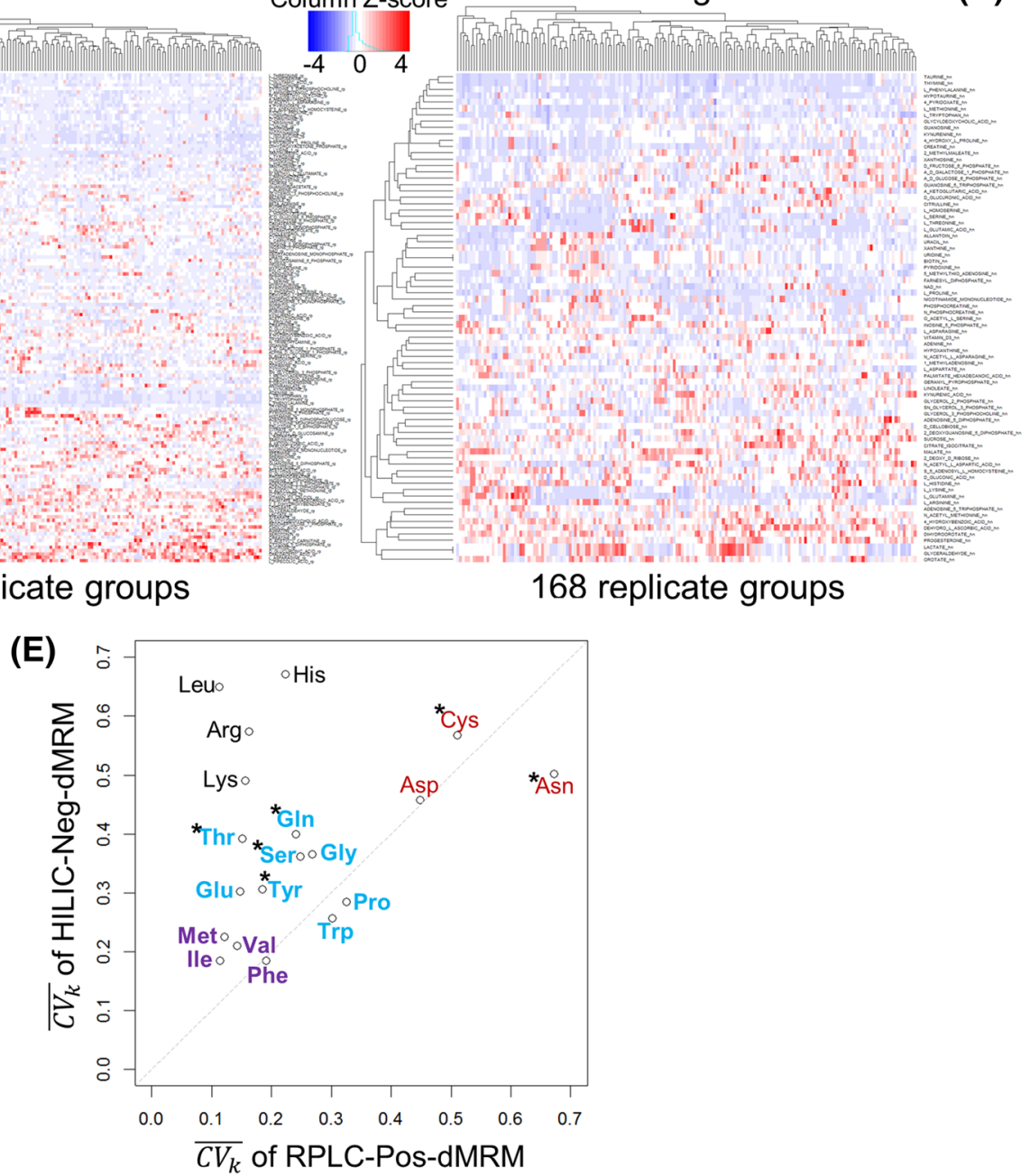

methods. The top metabolites with reliable measurements across most of the experiments include guanosine, 5-methlythio-adenosine, phosphocreatine, xanthine, proline, taurine, $\mathrm{NAD}+$, and methionine, among others. On the other hand, linoleate, glucuronic acid, 4-hydroxy-L-proline, dehydro-Lascorbic acid, and acetylcholine, among others show inconsistent measurements between the two methods, indicating that their retention mechanisms or ionization efficiency may 
४Fig. 3 Replicate-group CV analysis. a Distributions of replicategroup CVs of the RPLC-Pos-dMRM and HILIC-Neg-dMRM methods. There are 28,765 CV values in RPLC-Pos-dMRM and 22,069 $\mathrm{CV}$ values in HILIC-Neg-dMRM from all 183 replicate groups for 220 and 228 metabolites, respectively. Summary statistics of all replicate-group CVs from the two methods are shown in the inset. b The ordered distributions of $\left\{\overline{C V_{k}}\right\}$ for individual metabolites from the RPLC-Pos-dMRM and HILIC-Neg-dMRM methods. The top 5 and bottom 5 metabolites at the two tails are listed from each method. c Heatmap and hierarchical clustering of replicate-group CVs for 145 metabolites with missing values less than $30 \%$ across all replicate groups in the RPLC-Pos-dMRM method. d Heatmap and hierarchical clustering of replicate-group CVs for 77 metabolites with missing values less than $30 \%$ across all replicate groups in the HILIC-NegdMRM method. CVs are standardized by the column Z-score in $\mathbf{c}, \mathbf{d}$. e A scatter plot of $\left\{\overline{C V_{k}}\right\}$ of 19 amino acids shows a reproducibility trend and patterns as color coded as a guidance for 3 groups. The 15 amino acids show lower $\left\{\overline{C V_{k}}\right\}$ or better reproducibility in RPLC-PosdMRM, while the 4 amino acids do so in HILIC-Neg-dMRM. The six polar amino acids are indicated by asterisks. See also Supplementary Table S3 for all $\left\{\overline{C V_{k}}\right\}$ values in $\mathbf{b}$, $\mathbf{e}$ and the ordered lists of the 145 and 77 metabolites of $\mathbf{c}, \mathbf{d}$

greatly differ between the two methods from experiment to experiment on different days.

To further examine the 47 metabolites measured by the two methods, we next analyzed $\left\{\overline{C V_{k}}\right\}$ as in the previous section. Figure $4 \mathrm{~b}$ shows a distribution of $\left\{\overline{C V_{k}}\right\}$, where those

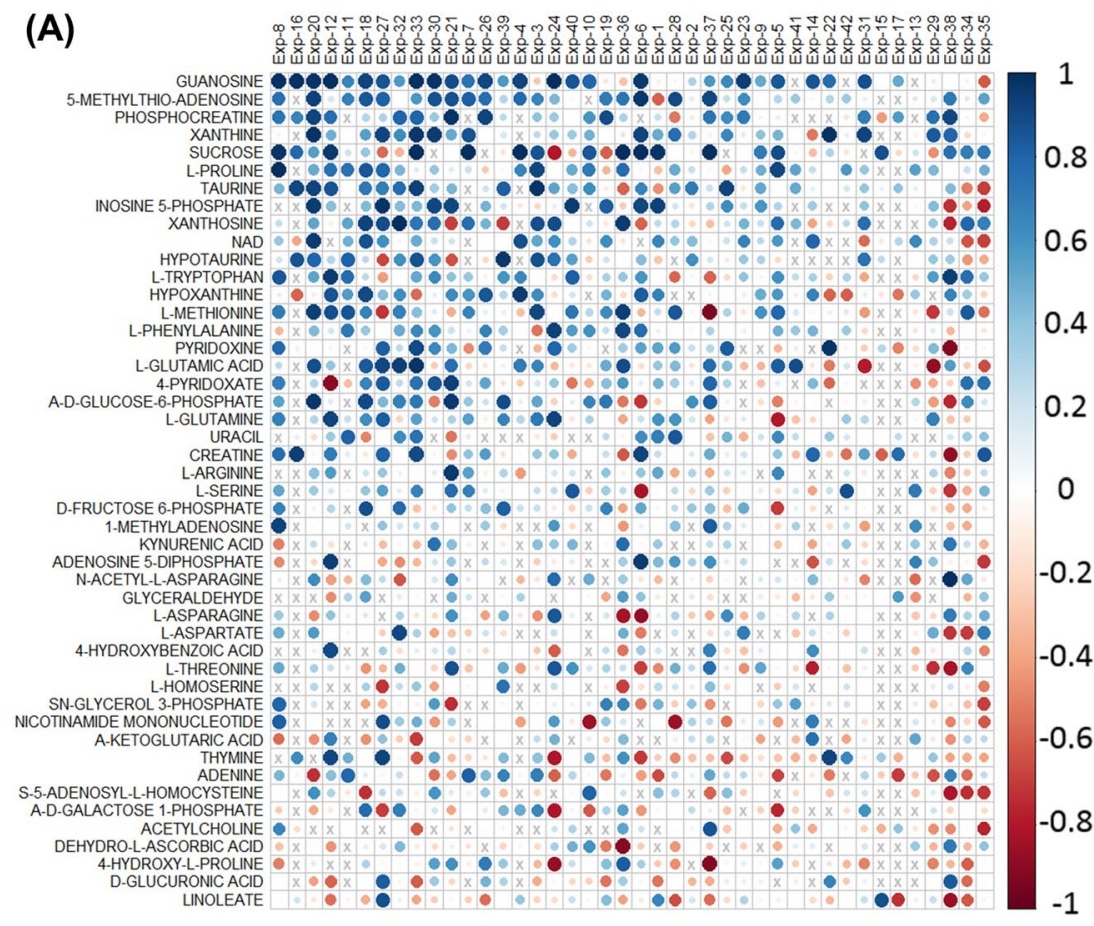

metabolites on the left with smaller $\overline{C V_{k}}$ are more reproducible and hence reliable across the 42 datasets by both methods on average. The top seven metabolites are methionine, 4-hydroxy-L-proline, phenylalanine, taurine, glutamic acid, hypotaurine, and NAD+ with $\overline{C V_{k}}<0.3$. We also calculated the average of $\left\{\overline{C V_{k}}\right\}$ in each of the two methods. The scatter plot in Fig. 4c shows a distribution of $\left\{\overline{C V_{k}}\right\}$ from the two methods for all 47 metabolites. Given our reference value of $\overline{C V_{k}}=0.4$, there are 15 metabolites with $\overline{C V_{k}}<0.4$ in both methods that we deem reproducible across diverse conditions. The three most reproducible metabolites from both methods are methionine, phenylalanine, and taurine. Phenylalanine was among the top 20 metabolites of high average abundance as discussed above. There are several metabolites which are reliable in either method alone based on a threshd of $\overline{C V_{k}}=0.4$. The RPLC-Pos-dMRM measurements are more reliable for 35 metabolites, whereas the HILIC-Neg-dMRM measurements are more reliable for 17 metabolites.
Fig. 4 RPLC-HILIC correlation analysis. a Heatmap of all RPLCHILIC Pearson correlation coefficients for 47 metabolites that were measured in at least $70 \%$ of all 42 experiments. The metabolites were sorted by the average correlation across all the experiments and the columns were sorted by the average correlation across all the metabolites. The size of the circles is proportional to absolute values of correlation coefficients. Larger circles in darker blues indicate good correlation between the RPLC-Pos-dMRM and HILIC-Neg-dMRM
(B)

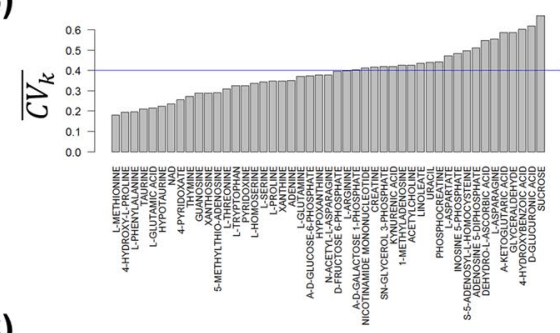

(C)

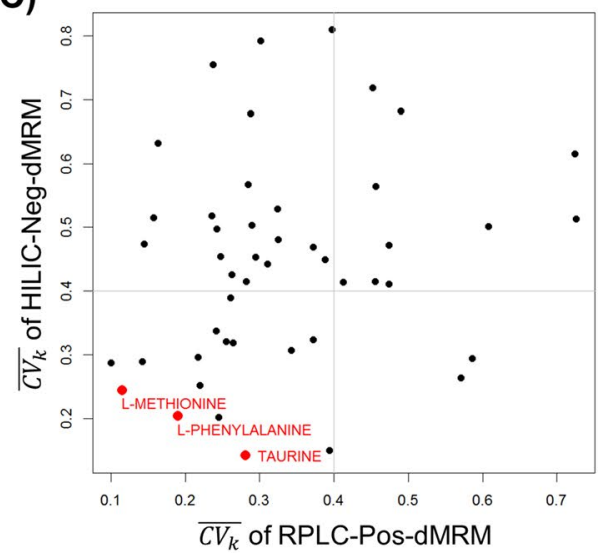

measurements. b Distribution of $\left\{\overline{C V_{k}}\right\}$ across the 42 datasets in both RPLC-Pos-dMRM and HILIC-Neg-dMRM methods. c A scatter plot of $\left\{\overline{C V_{k}}\right\}$ from the RPLC-Pos-dMRM and HILIC-Neg-dMRM measurements for all 47 metabolites. The three most reproducible metabolites by both methods are shown in red. See also Supplementary Table S4 for the list of 47 metabolites along with $\left\{\overline{C V_{k}}\right\}$ in both methods 


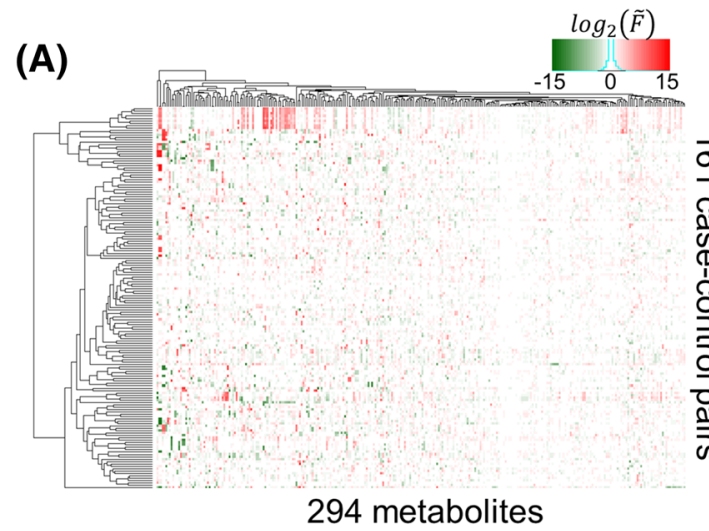

(RPLC-Pos-dMRM and HILIC-Neg-dMRM)

(C)

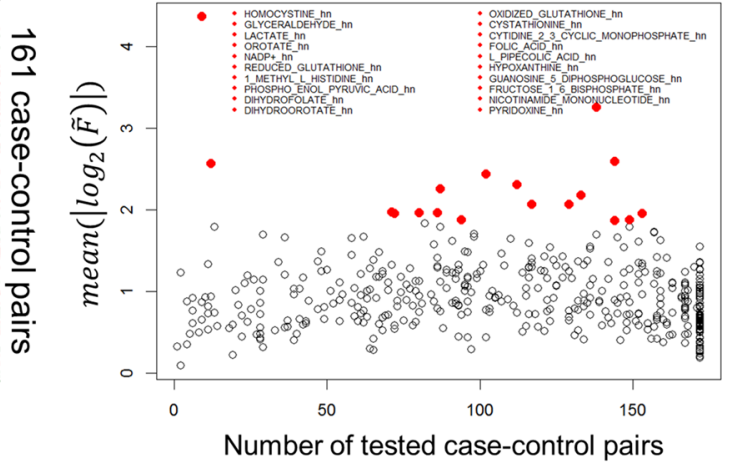

(E)

(D)
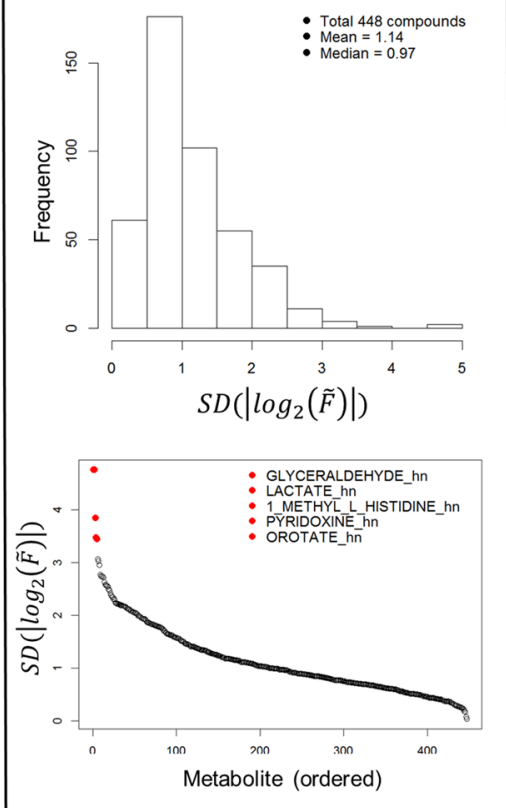
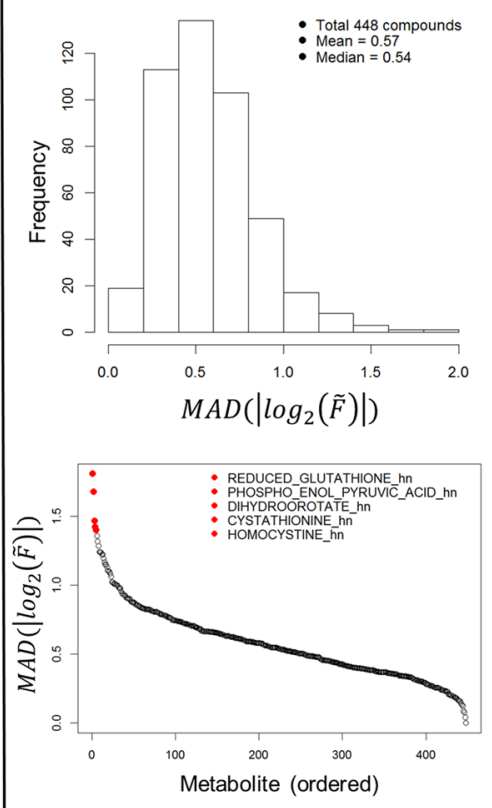

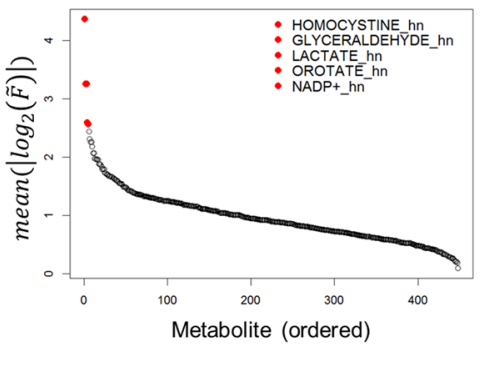

(F)

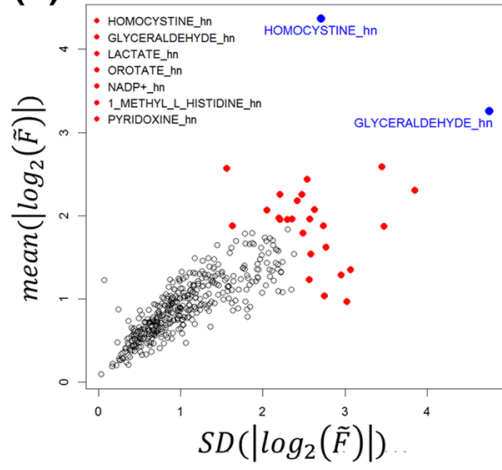

\subsection{Analysis of abundance fold changes for effect size and variability}

To study the directionality and magnitude of metabolite changes upon experimental perturbation, we analyzed fold

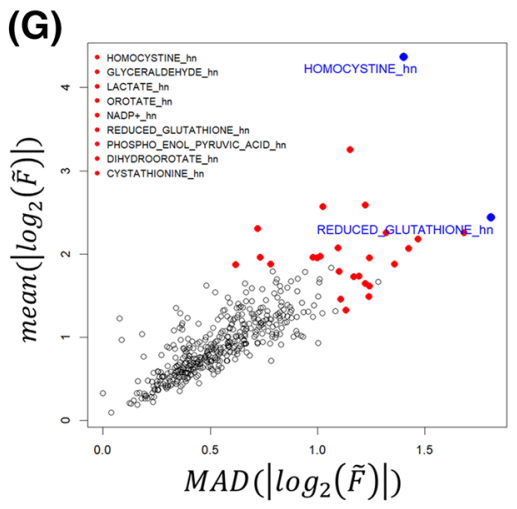

changes (FC) of metabolite abundance across all case-control condition group pairs. For all case-control pairs and each metabolite, we calculated the median $(\tilde{m})$ of normalized abundance values in each group of replicates, i.e., $\tilde{m}_{\text {case }}$ and $\tilde{m}_{\text {control }}$, and the fold change $(\tilde{F})$ of the medians, 
4Fig. 5 Abundance fold change analysis. a Heatmap of $\log _{2}(\tilde{F})$ for 294 metabolites and 161 case-control sample pairs. b A histogram of all average fold-change magnitudes $\left(=\operatorname{mean}\left(\left|\log _{2}(\tilde{F})\right|\right)\right.$; effect size $)$ and an ordered distribution of the average magnitudes. The top 20 metabolites are listed. c A scatter plot of the average fold-change magnitudes and the numbers of tested case-control pairs. d Effect-size variability in terms of the standard deviation (SD). A histogram of SDs of the effect sizes and an ordered SD distribution. The top five metabolites are listed. e Effect-size variability in terms of the median absolute deviation (MAD). A histogram of MADs of the effect sizes and an ordered MAD distribution. The top five metabolites are listed. f A scatter plot of the average effect sizes and the SD variability. The union of the top five metabolites from $\mathbf{b}, \mathbf{d}$ are listed. $\mathbf{g}$ A scatter plot of the average effect sizes and the MAD variability. The union of the top five metabolites from $\mathbf{b}$, e are listed. See Supplementary Table S5 for the full fold-change dataset

$\tilde{F}=\tilde{m}_{\text {case }} / \tilde{m}_{\text {control }}$. Then, we calculated the average of absolute values of $\log _{2}(\tilde{F})$ for all case-control pairs for each metabolite, mean $\left(\left|\log _{2}(\tilde{F})\right|\right)$, which represents an average magnitude of the fold change in the case group relative to the control group for that metabolite. The magnitude or absolute value of $\log _{2}(\tilde{F})$ is also called the effect size.

There are a total of 448 metabolites in 176 case-control pairs from both RPLC-Pos-dMRM and HILIC-NegdMRM methods. There are $37.3 \%$ missing values in the 448 by 176 data matrix. A missing value occurs when no measurement was made in either a case or control condition. For a hierarchical clustering of the full data, we removed those metabolites and case-control pairs that have more than $50 \%$ missing values across all case-control pairs and all metabolites, respectively. This left us with 294 metabolites and 161 case-control pairs with $15.8 \%$ missing values. Figure 5a shows a heatmap of the 294 by 161 data matrix of $\log _{2}(\tilde{F})$ values along with hierarchical clustering dendrograms on both axes. The full 448 by 176 data matrix of fold changes gives us a histogram of all average fold-change magnitudes defined by mean $\left(\left|\log _{2}(\tilde{F})\right|\right)$ and their ordered distribution in Fig. 5b. The average fold-change magnitudes for most metabolites are less than 2 (the peak of the histogram) and the top 20 metabolites with the highest average effect sizes are mostly involved in glycolysis, redox, pyrimidine, and methionine/ cysteine/folate metabolism. Homocystine shows the largest average FC magnitude of more than 20, and lactate/ glyceraldehyde the second largest of more than 9 . We note that lactate and glyceraldehyde were not distinguishable in our HILIC-Neg-dMRM method with identical data. Most of the top 20 metabolites were measured in more than 70 case-control pairs, except homocystine in nine pairs in the HILIC method and NADP in 12 pairs in the HILIC method (Fig. 5c). In addition, we calculated the standard deviation (SD) of the effect sizes for each metabolite to examine the effect-size variability in all case-control pairs for that metabolite. Figure 5d shows a histogram of the SDs and an ordered SD distribution. The SDs for most metabolites are less than 1 and there are seven metabolites with $\mathrm{SD}>3$ including lactate/glyceraldehyde and orotate. We also performed an analysis of median absolute deviation (MAD) to complement the SD analysis. Figure 5e shows a MAD histogram and an ordered MAD distribution. The top 20 most variable metabolites with the highest MADs are involved in glycolysis, redox, pyrimidine, energy, and methionine/cysteine/folate metabolism. Figure 5f, g show good positive correlations between the average effect sizes and the SD and MAD variability, respectively. Homocystine, lactate/glyceraldehyde, orotate, GSH, and dihydrofolate have both the highest average effect size and the largest variability by both SD and MAD among the top 20, which suggests that they are most dynamic and responsive metabolites across this diverse array of conditions.

We note that we have not performed analysis of batch effects in this study because it is challenging to analyze and model batch effects in all 42 datasets in a reasonable way. Any attempt to correct batch effects globally may introduce mathematical artifacts into certain data points locally, making biological interpretations more complex or unreasonable across all datasets. We consider the analysis of batch effects an independent topic for a future study. Therefore, our analysis and results should be interpreted within the constraints of this limitation.

\section{Conclusions}

We performed LC-MS/MS targeted metabolomics to generate 42 datasets in a wide range of experiments and sample types from the same platforms with both RPLC-PosdMRM and HILIC-Neg-dMRM methods. We carried out a series of computational and statistical analyses to systematically assess data quality of both methods in biological replicate groups to identify more reliable measurements in each method. We find that the RPLC-Pos-dMRM method tends to generate more reproducible measurements than the HILIC-Neg-dMRM method, including polar amino acids. On the other hand, several metabolites are measured reliably in both methods such as methionine, phenylalanine, and taurine. Phenylalanine is among the most abundant metabolites across all samples on average along with acetylcarnitine, cystine, arginine, lactate/glyceraldehyde, UDP-GlcNAc, AMP, GSH, and acetyl-CoA. We also characterized global abundance changes and variability in case-control groups to identify most dynamic and variable metabolites across heterogeneous conditions, such as homocystine, lactate/ glyceraldehyde, and GSH. Those metabolites are involved in cysteine metabolism (taurine, cystine, homocystine), glycolysis (glyceraldehyde, lactate), hexosamine biosynthesis 
(UDP-GlcNAc), redox balance (GSH), fatty acids metabolism (acetylcarnitine, acetyl-CoA), and the TCA cycle (acetyl-CoA). This might suggest that they tend to play more significant roles than others in the associated metabolic pathways under many different conditions. For example, lactate, taurine, methionine, and acetylcarnitine were recently found to be more frequently differentially abundant in tumors compared to normal tissues across different cancer types (Reznik et al. 2018). Our study offers a systematic guideline and a reference point in targeted metabolomics analysis by either RPLC-Pos-dMRM or HILIC-Neg-dMRM method for more reliable biological interpretations without sample-dependent optimization of the LC-MS system.

\section{Supplementary material}

The Supplementary Material is provided in a compressed zip file containing the following materials. A list of metabolites measured in RPLC-Pos-dMRM and HILIC-Neg-dMRM methods is available in the Supplementary Table (MS Excel file) along with their formulae, precursor ion mass, product ion mass, RTs, RT window, and CAS numbers (Excel spreadsheet, "Methods"). All source data used for Figs. 1 to 5 are available in the Supplementary Table (Excel spreadsheets, "Table S1" to "Table S5"). The raw abundance data of peak areas are available in the Supplementary Table (Excel spreadsheet, "Raw data"). All analysis codes in R and associated data are available in the file directory, "codes".

Acknowledgements The authors would like to thank all of anonymous collaborators who provided the samples used in this study and Drs. Garrison Birch, Steven Fischer, and Maureen Kachman for their critical comments and suggestions. The authors would like to also thank the reviewer, Dr. Curt Fischer, and 2 other anonymous reviewers for their constructive comments and suggestions. D.M.K is supported by the University of Michigan's Program in Chemical Biology Graduate Assistance in Areas of National Need (GAANN) award. C.A.L. was supported by a Pancreatic Cancer Action Network/AACR Pathway to Leadership award (13-70-25-LYSS); Dale F. Frey Award for Breakthrough Scientists from the Damon Runyon Cancer Research Foundation (DFS-09-14); Junior Scholar Award from The V Foundation for Cancer Research (V2016-009); Kimmel Scholar Award from the Sidney Kimmel Foundation for Cancer Research (SKF-16-005); a 2017 AACR NextGen Grant for Transformative Cancer Research (17-2001-LYSS); and UMCCC Core Grant (P30 CA046592). Metabolomics studies performed at the University of Michigan were supported by NIH grant DK097153, the Charles Woodson Research Fund and the UM Pediatric Brain Tumor Initiative.

Author contributions HL conceived the idea, designed the algorithms and pipelines, performed computational and statistical analyses, interpreted the results, and wrote a draft of the manuscript. DMK and PS prepared the samples and helped with raw data processing. LZ performed LC-MS analysis including raw data generation. DMK and LZ contributed to writing the manuscript. CAL conceived the idea, interpreted the results, contributed to writing the manuscript, and supervised the project. All authors read and approved the manuscript.

\section{Compliance with ethical standards}

Conflict of interest Costas A. Lyssiotis is an inventor on patents pertaining to Kras regulated metabolic pathways, redox control pathways in pancreatic cancer, and targeting GOT1 as a therapeutic approach. Ho-Joon Lee, Daniel M. Kremer, Peter Sajjakulnukit and Li Zhang declare that they have no conflict of interest.

Ethical approval This article does not contain any studies with human and/or animal participants performed by any of the authors.

Open Access This article is distributed under the terms of the Creative Commons Attribution 4.0 International License (http://creativecommons.org/licenses/by/4.0/), which permits unrestricted use, distribution, and reproduction in any medium, provided you give appropriate credit to the original author(s) and the source, provide a link to the Creative Commons license, and indicate if changes were made.

\section{References}

Buszewski, B., \& Noga, S. (2012). Hydrophilic interaction liquid chromatography (HILIC) - A powerful separation technique. Analytical and Bioanalytical Chemistry, 402, 231-247.

Carobene, A., Braga, F., Roraas, T., Sandberg, S., \& Bartlett, W. A. (2013). A systematic review of data on biological variation for alanine aminotransferase, aspartate aminotransferase and gammaglutamyl transferase. Clinical Chemistry and Laboratory Medicine, 51, 1997-2007.

Chong, J., Soufan, O., Li, C., Caraus, I., Li, S., Bourque, G., et al. (2018). MetaboAnalyst 4.0: Towards more transparent and integrative metabolomics analysis. Nucleic Acids Research, 46, W486-W494.

Cubbon, S., Antonio, C., Wilson, J., \& Thomas-Oates, J. (2010). Metabolomic applications of HILIC-LC-MS. Mass Spectrometry Reviews, 29, 671-684.

Griffiths, W. J., Koal, T., Wang, Y., Kohl, M., Enot, D. P., \& Deigner, H. P. (2010). Targeted metabolomics for biomarker discovery. Angewandte Chemie, 49, 5426-5445.

Halbrook, C. J., Pontious, C., Kovalenko, I., Lapienyte, L., Dreyer, S., Lee, H. J., et al. (2019). Macrophage released pyrimidines inhibit gemcitabine therapy in pancreatic cancer. Cell Metabolism, 29(6), 1390-1399.

Halbrook, C. J., Pontious, C., Lee, H.-J., Kovalenko, I., Zhang, Y., Lapienyte, L., et al. (2018). Macrophage released pyrimidines inhibit gemcitabine therapy in pancreatic cancer. Cell Metabolism, 29(6), 1390-1399.

Hao, Z., Xiao, B., \& Weng, N. (2008). Impact of column temperature and mobile phase components on selectivity of hydrophilic interaction chromatography (HILIC). Journal of Separation Science, 31, 1449-1464.

Ivanisevic, J., Zhu, Z. J., Plate, L., Tautenhahn, R., Chen, S., O’Brien, P. J., et al. (2013). Toward 'omic scale metabolite profiling: A dual separation-mass spectrometry approach for coverage of lipid and central carbon metabolism. Analytical Chemistry, 85, 6876-6884.

Johnson, C. H., Ivanisevic, J., \& Siuzdak, G. (2016). Metabolomics: Beyond biomarkers and towards mechanisms. Nature Reviews Molecular Cell Biology, 17, 451-459.

Kirpich, A. S., Ibarra, M., Moskalenko, O., Fear, J. M., Gerken, J., Mi, X., et al. (2018). SECIMTools: A suite of metabolomics data analysis tools. BMC Bioinformatics, 19, 151. 
Li, B., Tang, J., Yang, Q., Li, S., Cui, X., Li, Y., et al. (2017). NOREVA: Normalization and evaluation of MS-based metabolomics data. Nucleic Acids Research, 45, W162-w170.

Lu, W., Bennett, B. D., \& Rabinowitz, J. D. (2008). Analytical strategies for LC-MS-based targeted metabolomics. Journal of Chromatography, B: Analytical Technologies in the Biomedical and Life Sciences, 871, 236-242.

Patti, G. J., Yanes, O., \& Siuzdak, G. (2012). Innovation: Metabolomics: The apogee of the omics trilogy. Nature Reviews Molecular Cell Biology, 13, 263-269.

Reznik, E., Luna, A., Aksoy, B. A., Liu, E. M., La, K., Ostrovnaya, I., et al. (2018). A landscape of metabolic variation across tumor types. Cell Systems, 6, 301-313.e303.

Rhoades, S. D., \& Weljie, A. M. (2016). Comprehensive optimization of LC-MS metabolomics methods using design of experiments (COLMeD). Metabolomics, 12, 183.

Rojo, D., Barbas, C., \& Ruperez, F. J. (2012). LC-MS metabolomics of polar compounds. Bioanalysis, 4, 1235-1243.

Schofield, H. K., Zeller, J., Espinoza, C., Halbrook, C. J., Del Vecchio, A., Magnuson, B., et al. (2018). Mutant p53R270H drives altered metabolism and increased invasion in pancreatic ductal adenocarcinoma. JCI Insight, 3, 97422.

Sousa, C. M., Biancur, D. E., Wang, X., Halbrook, C. J., Sherman, M. H., Zhang, L., et al. (2016). Pancreatic stellate cells support tumour metabolism through autophagic alanine secretion. Nature, 536, 479-483.

Svoboda, L. K., Teh, S. S. K., Sud, S., Kerk, S., Zebolsky, A., Treichel, S., et al. (2018). Menin regulates the serine biosynthetic pathway in Ewing sarcoma. The Journal of Pathology, 245, $324-336$
Tang, D. Q., Zou, L., Yin, X. X., \& Ong, C. N. (2016). HILIC-MS for metabolomics: An attractive and complementary approach to RPLC-MS. Mass Spectrometry Reviews, 35, 574-600.

van den Berg, R. A., Hoefsloot, H. C., Westerhuis, J. A., Smilde, A. K., \& van der Werf, M. J. (2006). Centering, scaling, and transformations: Improving the biological information content of metabolomics data. BMC Genomics, 7, 142.

Wanichthanarak, K., Fan, S., Grapov, D., Barupal, D. K., \& Fiehn, O. (2017). Metabox: A toolbox for metabolomic data analysis, interpretation and integrative exploration. PLOS ONE, 12, e0171046.

Wishart, D. S. (2016). Emerging applications of metabolomics in drug discovery and precision medicine. Nature Reviews Drug Discovery, 15, 473-484.

Xie, B., Wang, Y., Jones, D. R., Dey, K. K., Wang, X., Li, Y., et al. (2018). Isotope labeling-assisted evaluation of hydrophilic and hydrophobic liquid chromatograph-mass spectrometry for metabolomics profiling. Analytical Chemistry, 90, 8538-8545.

Yuan, M., Breitkopf, S. B., Yang, X., \& Asara, J. M. (2012). A positive/negative ion-switching, targeted mass spectrometry-based metabolomics platform for bodily fluids, cells, and fresh and fixed tissue. Nature Protocols, 7, 872-881.

Zhou, J., \& Yin, Y. (2016). Strategies for large-scale targeted metabolomics quantification by liquid chromatography-mass spectrometry. Analyst, 141, 6362-6373.

Publisher's Note Springer Nature remains neutral with regard to jurisdictional claims in published maps and institutional affiliations.

\section{Affiliations}

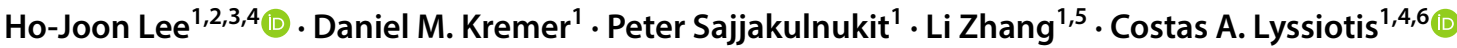

1 Department of Molecular and Integrative Physiology, University of Michigan Medical School, Ann Arbor, MI 48109, USA

2 Center for Computational Medicine and Bioinformatics, University of Michigan Medical School, Ann Arbor, MI 48109, USA

3 Michigan Institute for Data Science, University of Michigan, Ann Arbor, MI 48109, USA
4 Rogel Cancer Center, University of Michigan, Ann Arbor, MI 48109, USA

5 Michigan Regional Comprehensive Metabolomics Resource Core, University of Michigan, Ann Arbor, MI 48109, USA

6 Department of Internal Medicine, Division of Gastroenterology and Hepatology, University of Michigan Medical School, Ann Arbor, MI 48109, USA 